\title{
A Domain Specific Language for Composable Memory Transactions in Java
}

\author{
André Rauber Du Bois and Marcos Echevarria \\ PPGInf - Programa de Pós-Graduação em Informática, \\ Universidade Católica de Pelotas \\ CEP: 96010-000, Pelotas-RS, Brazil \\ \{dubois, marcosge\}@ucpel.tche.br
}

\begin{abstract}
In this paper we present CMTJava, a domain specific language for composable memory transactions [7] in Java. CMTJava provides the abstraction of transactional objects. Transactional objects have their fields accessed only by special get and set methods that are automatically generated by the compiler. These methods return transactional actions as a result. A transactional action is an action that, when executed, will produce the desired effect. Transactional actions can only be executed by the atomic method. Transactional actions are first class values in Java and they are composable: transactions can be combined to generate new transactions. The Java type system guarantees that the fields of transactional objects will never be accessed outside a transaction. CMTJava supports the retry and orElse constructs from STM Haskell. To validate our design we implemented a simple transactional system following the description of the original Haskell system. CMTJava is implemented as a state passing monad using BBGA closures, a Java extension that supports closures in Java.
\end{abstract}

\section{Introduction}

Multi-core machines are now available everywhere. The traditional way to program these machines using threads, shared memory and locks for synchronization is difficult and has many pitfalls [1622 24]. A promising approach to program multi-core machines is to use software transactional memory (STM). In this approach, accesses to shared memory are performed inside transactions, and a transaction is guaranteed to be executed atomically with respect to other concurrently executed transactions. Transactions can be implemented using optimistic concurrency: all transactions are executed concurrently with no synchronization, all writes and reads to shared memory are performed in a transaction log. When a transaction finishes, it validates its $\log$ to check if it has seen a consistent view of memory, and if so, its changes are committed to memory. If validation fails the $\log$ is discarded and the transaction is executed again with a fresh log. With atomic transactions the programmer still has to worry when critical sections should begin and end. But although having to delimit critical sections, the programmer does not need to worry about a locking protocol, that can induce problems like deadlocks. 
In this paper, we present CMTJava, a domain specific language to write composable memory transactions in Java. CMTJava brings the idea of composable memory transactions in the functional language Haskell [7] to a object oriented context.

The main characteristics of the CMTJava system are:

- CMTJava provides the abstraction of Transactional Objects. Transactional objects have their fields accessed only by special get and set methods that are automatically generated by the compiler. These methods return transactional actions as a result. A transactional action is an action that, when executed, will produce the desired effect. Java's type system guarantees that the fields of transactional objects can only be accessed inside transactions. Transactions can only executed with the atomic method. The atomic method takes a transaction as an argument and executes it atomically with respect to all other memory transactions. As transactions can not be executed outside a call to atomic, properties like atomicity (the effects of a transaction must be visible to all threads all at once) and isolation (during the execution of a transaction, it can not be affected by other transactions) are always manteined.

- Transactional actions are first class values in Java, they can be passed as arguments to methods and can be returned as the result of a method call. Transactions are composable: they can be combined to generate new transactions. Transactions are composed using STM blocks (Section 2), and we also support the retry construct, that allows possibly-blocking transactions to be composed sequentially and the orElse construct that allows transactions to be composed as alternatives.

- Transactions are implemented as a state passing monad (Section 3.3). Most of the simplicity of the implementation comes from the fact that we use a Java extension for closures to implement CMTJava. Hence, this paper is also a case support for closures in Java 7 . We describe translation rules that translate CMTJava to pure Java + closures. Although the system was implemented in Java, the ideas could also be applied to any object oriented language that supports closures, e.g., C\#.

The paper is organized as follows. First, in Section 2, CMTJava is presented through simple examples. Section 3 , describes the implementation of the language primitives using a state passing monad. A simple prototype implementation of transactions is also described. Section 4 discusses related work and finally Section 5 presents conclusions and directions for future work.

\section{Examples}

In this section we present simple examples with the objective of describing how to program with CMTJava. 


\subsection{The Dinning Philosophers}

In this section, an implementation of the dinning philosophers problem is given using the CMTJava DSL. The first class to be defined is Fork:

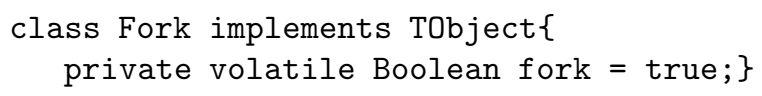

The Fork class has only one field and Java's volatile keyword is used to guarantee that threads will automatically see the most up-to-date value in the field. The TObject interface works as a hint to the compiler, so it will generate automatically the code needed to access this class in transactions. It also generates two methods to access each field of the class. For the Fork class it will generate the following methods:

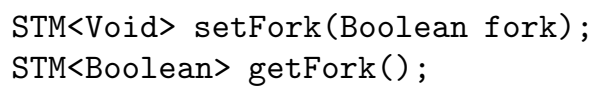

The setFork and getFork methods are the only way to access the fork field. A value of type STM $\angle A>$ represents a transactional action that when executed will produce a value of type A.

We can now define the Philosopher class as in Figure 1 The STM $\{\ldots\}$ block works like the do notation in STM Haskell [7]: it is used to compose transactions. The notation $\operatorname{STM}\left\{a_{1} ; \ldots ; a_{n}\right\}$ constructs a STM action by glueing together smaller actions $a_{1} ; \ldots ; a_{n}$ in sequence. Using STM blocks it is

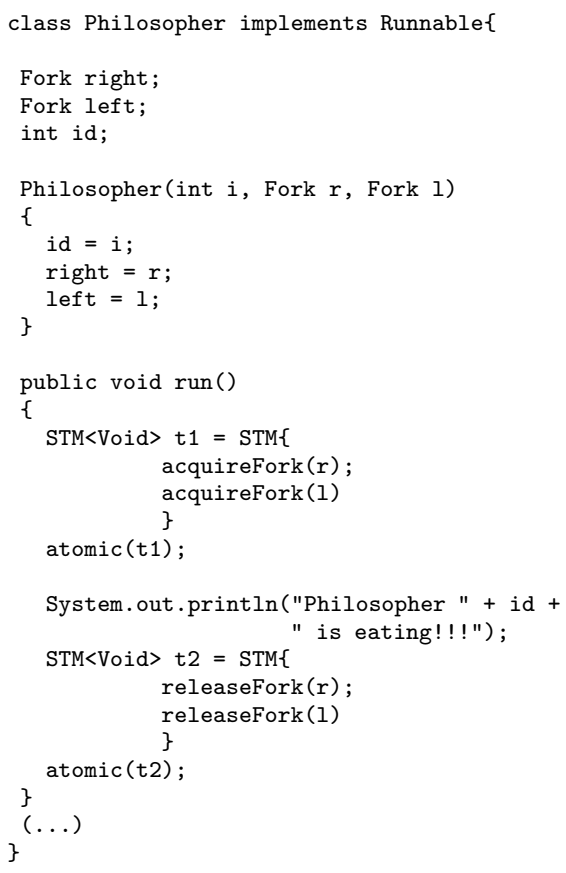

Fig. 1. The Philosopher class 
possible to implement the STM<Void> acquireFork(Fork $f$ ) and STM<Void> releaseFork(Fork $f$ ) methods that are missing on Figure 1 .

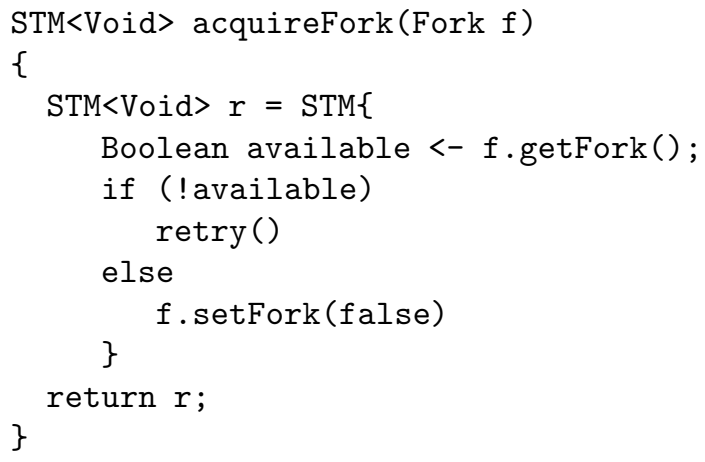

The acquireFork method first checks the current state of the Fork using the getFork () method. If the fork is not available it blocks the transaction by calling retry. If the fork was available, it is set to false, i.e., not available. The call to retry will block the transaction, i.e., the transaction will be aborted and restarted from the beginning. The transaction will not be re-executed until at least one of the fields of the TObjects that it has accessed is written by another thread. In the case of the acquireFork method, when it calls retry the philosopher will be suspended until a neighbor philosopher puts the fork back in the table. The variables created inside a transactional action are single assignment variables. These variables are used only to carry the intermediate state of the transaction being constructed and do not need to be logged by the runtime system supporting transactions. To emphasize that these variables are different than the ordinary Java variable a different symbol for assignment is used $((<-)$.

The releaseFork method has a simple implementation:

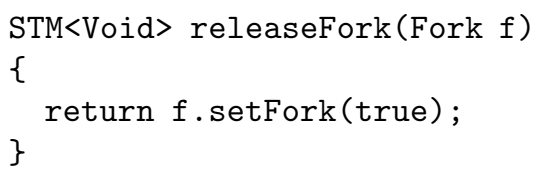

It returns a transaction that when executed will set the fork field to true. When a philosopher calls releaseFork it already holds the fork so there is no need to check its current state.

\subsection{The orElse Method}

CMTJava also provides the orElse method that allows the composition of transactions as alternatives

public static $<\mathrm{A}>\mathrm{STM}<\mathrm{A}>$ orElse $(\mathrm{STM}<\mathrm{A}>\mathrm{t1}, \mathrm{STM}<\mathrm{A}>\mathrm{t} 2)$ 
orElse takes two transactions as arguments and returns a new transaction. The transaction

$$
\operatorname{orElse}(t 1, \mathrm{t} 2) \text {; }
$$

will first execute $t 1$; if it retries then $t 1$ is discarded and $t 2$ is executed. If $t 2$ also retries then the whole transaction will retry.

In Figure 2 a simple implementation of a class representing a bank account is given. Using orElse we could extend the Account class with the following method:

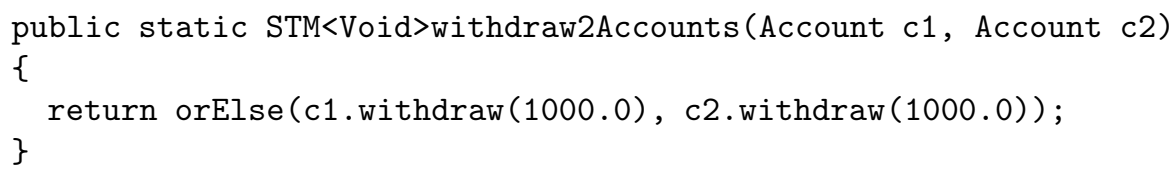

The withdraw2Accounts method first tries to withdraw 1000.0 from $\mathrm{c} 1$. If there is not enough money in that account it tries to withdraw the same amount from c2

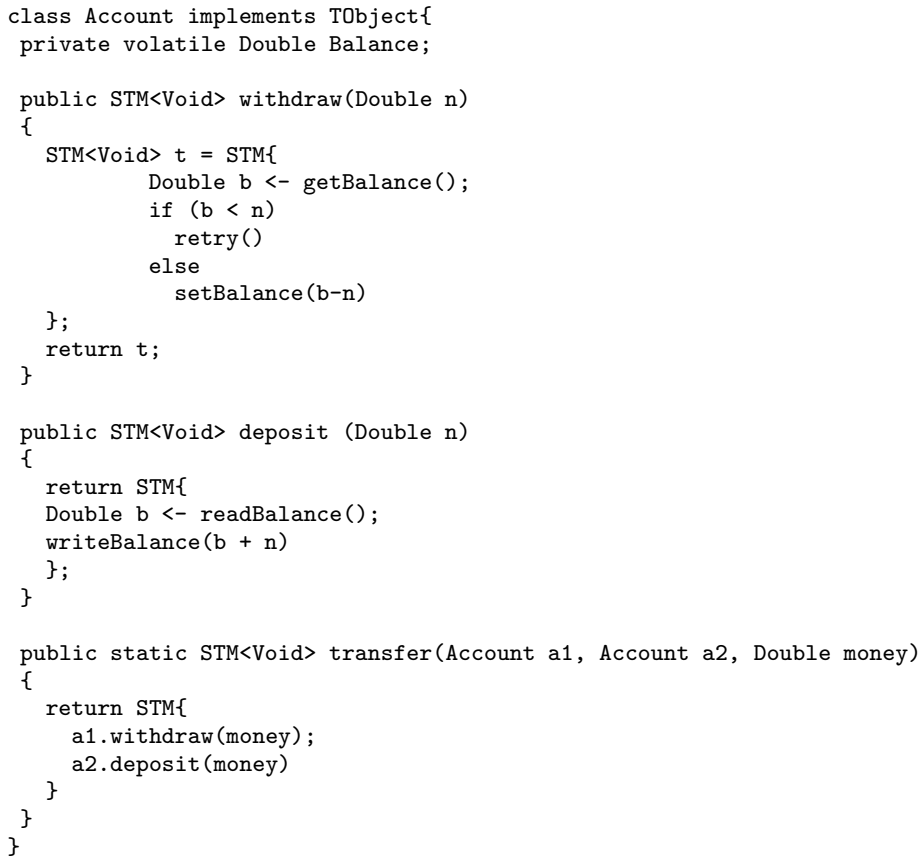

Fig. 2. The Account class 


\section{Implementation}

\subsection{Java Closures}

To implement CMTJava we used BGGA Closures, a Java extension that supports anonymous functions and closures [2]. This extension is a prototype implementation of a JSR proposal to add closures to Java 7 [3].

In BBGA, an anonymous function can be defined using the following syntax:

$\{$ formal parameters $=>$ statements expression $\}$

where formal parameters, statements and expression are optional. For example, \{ int $x=>x+1$ \} is a function that takes an integer and returns its value incremented by one. An anonymous function can be invoked using its invoke method:

String $\mathbf{S}=\{=>$ Hello!" $\}$.invoke();

An anonymous function can also be assigned to variables:

$\{$ int $\Rightarrow$ void $\}$ func $=\{$ int $x \Rightarrow$ System.out.println $(x)\}$;

The variable func has type $\{$ int $\Rightarrow>$ void , i.e., a function type meaning that it can be assigned to a function from int to void. Function types can also be used as types of arguments in a method declaration.

A closure is a function that captures the bindings of free variables in its lexical context:

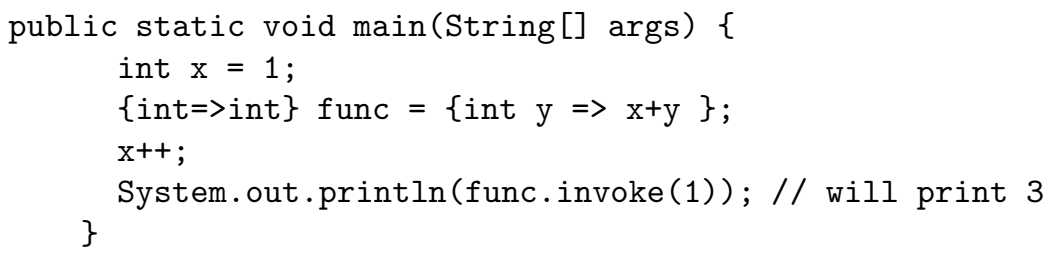

A closure can use variables of the enclosing scope even if this scope is not active at the time of closure invocation e.g., if a closure is passed as an argument to a method it will still use variables from the enclosing scope where it was created.

\subsection{Monads}

A monad is a way to structure computations in terms of values and sequences of computations using those values [1]. A monad is used to describe computations and how to combine these computations to generate new computations. For this reason monads are frequently used to embed domain specific languages in functional languages for many different purposes, e.g., I/O and concurrency [20], Parsers [13, controlling robots [19], and memory transactions [7. A monad can be implemented as an abstract data type that represents a container for a computation. These computations can be created and composed using three basic operations: bind, then and return. For any monad $\mathrm{m}$, these functions have the following type in Haskell: 


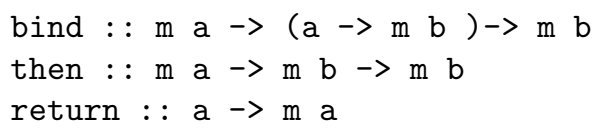

The type $\mathrm{m}$ a represents a computation in the monad $\mathrm{m}$ that when executed will produce a value of type a. The bind and then functions are used to combine computations in a monad. bind executes its first argument and passes the result to its second argument (a function) to produce a new computation. then takes two computations as arguments and produces a computation that will execute them one after the other. The return function creates a new computation from a simple value.

The next section presents the implementation of these three operations for the STM monad in Java.

\subsection{The STM Monad}

The monad for STM actions is implemented as a state passing monad. The state passing monad is used for threading a state through computations, where each computation returns an altered copy of this state. In the case of transactions, this state is the meta-data for the transaction, i.e., its log.

The STM class is implemented as follows:

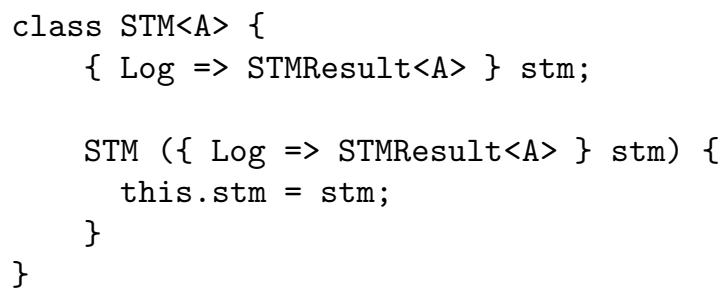

The STM class is used to describe transactions, and it has only one field: a function representing the transaction. A transaction STM $\angle A>$ is a function that takes a Log as an argument and returns a STMResul $t<A>$. The Log represents the current state of the transaction being executed and STMResult describes the new state of the transaction after its execution.

The STMResult $<\mathrm{A}>$ class has three fields:

class STMResult $<\mathrm{A}>\{$

A result;

Log newLog;

int state;

$(\ldots)$ 
the first field (result) is the result of executing the STM action, the second (newLog) is the resulting log, and the third is the current state of the transaction. Executing a STM action can put the transaction in one of two states: it is either VALID meaning the the transaction can continue, or the state can be set to RETRY meaning that the retry construct was called and the transaction must be aborted.

The bind method is used to compose transactional actions:

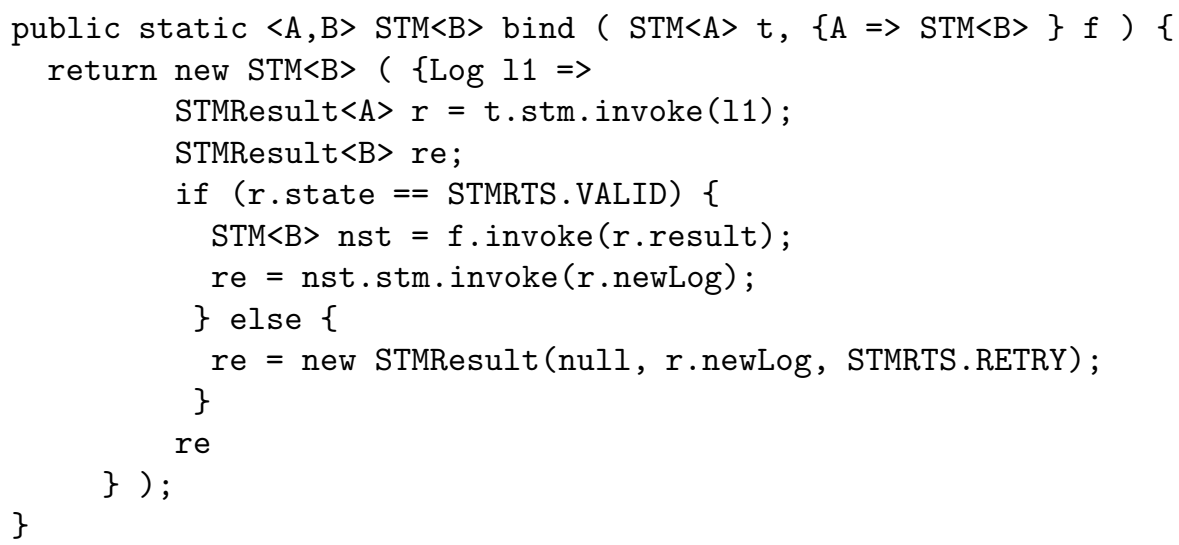

The bind method takes as arguments an STM $<A>$ action $t$ and a function $f$ of type $\{\mathrm{A} \Rightarrow \mathrm{STM}\langle\mathrm{B}>\}$ and returns as a result a new $\mathrm{STM}<\mathrm{B}>$ action. The objective of bind is to combine STM actions generating new actions. The resulting STM action takes a $\log (11)$ as an argument and invokes the $t$ action by passing the $\log$ to it (t.stm. invoke (11)). If the invocation of $t$ did not retry (i.e., its state is VALID) then $f$ is called generating the resulting STM $\angle B>$. Otherwise the execution flow is abandoned and bind returns a STMResult with the state set to RETRY.

The then method is implemented in a very similar away

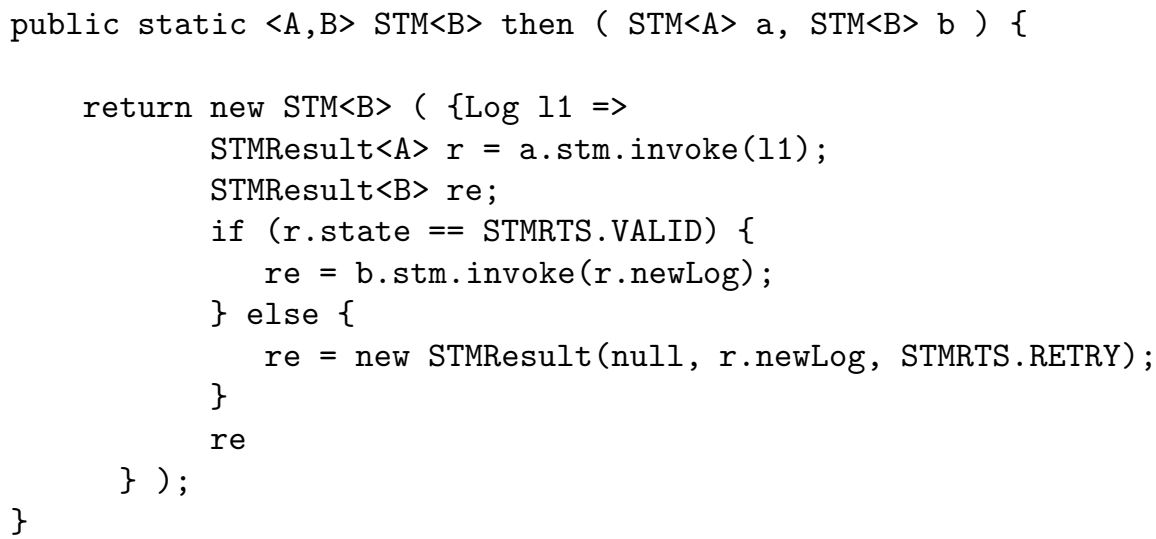

The then method is a sequencing combinator: it receives as arguments two STM actions and returns an action that will execute them one after the order. 
Finally, the stmreturn method is used to insert any object A into the STM monad:

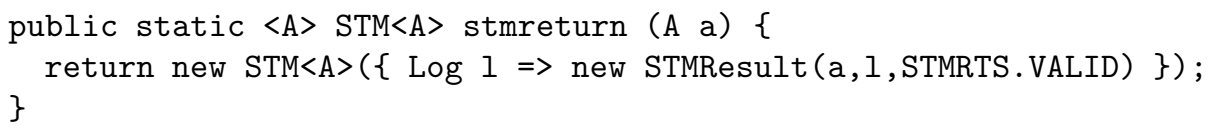

The stmreturn method is like Java's return for STM blocks. It takes an object as an argument and creates a simple transaction that returns this object as a result. It can also be used to create new objects inside a transaction. For example, the insert method returns a transaction that inserts a new element at the end of a linked list:

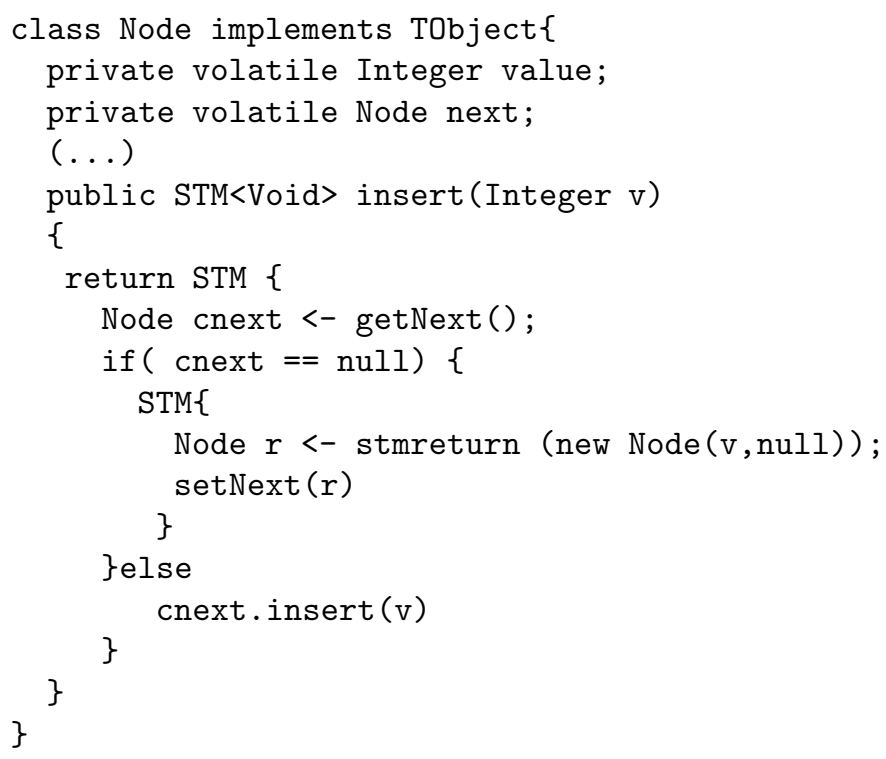

\subsection{STM Blocks}

STM blocks are translated into calls to bind and then using the translation rules given in Figure 3. Translation rules for STM blocks are very similar to the translation rules for the do notation described in the Haskell report [21].

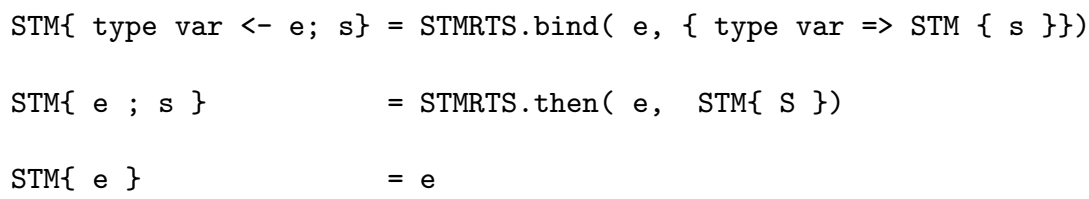

Fig. 3. Basic translation schemes for STM blocks 
For example, the following implementation of a deposit method:

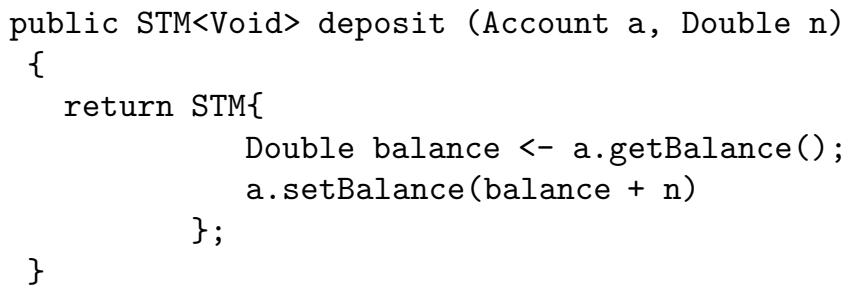

\subsection{Compiling Objects}

A class like Fork given in section 2.1 is compiled to the class in Figure 4, using the translation rules given in Appendix A.

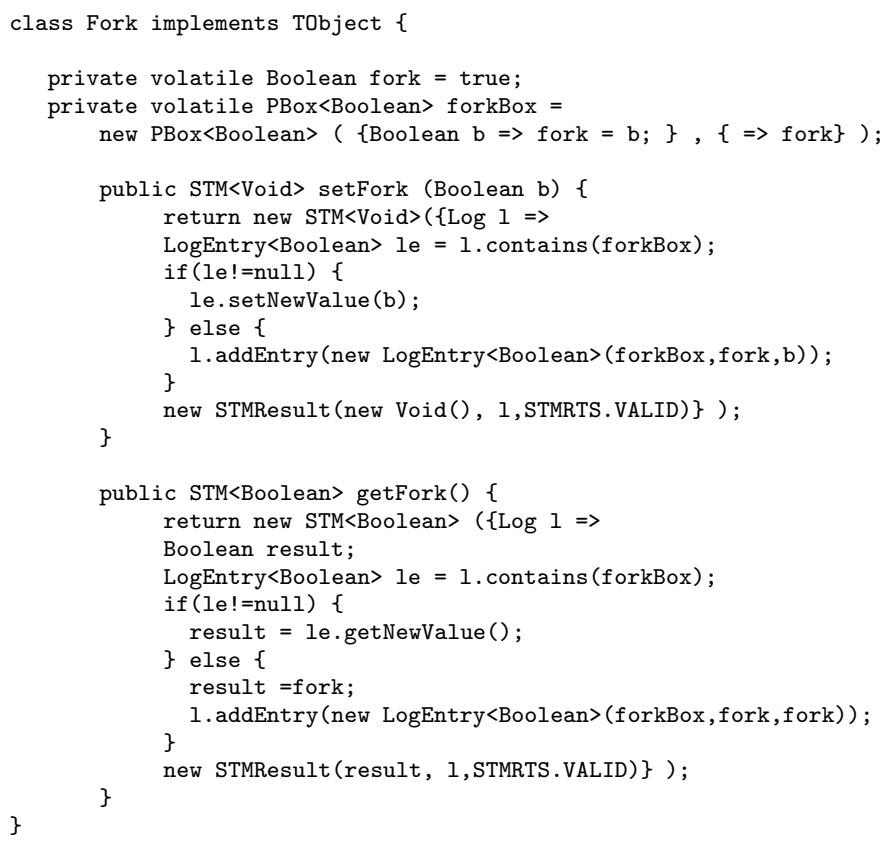

Fig. 4. The Fork class after compilation 
Every TObject has a PBox for each of its fields. A PBox works like a pointer to the field. The constructor of the PBox class takes two arguments: one is a function that updates the current value of a field and the other is a function that returns the current value of a field. This functions are used by atomic to commit a transaction (Section 3.8).

The setFork and getFork methods are used to access the fork field in a transaction. The setFork method first verifies if the PBox for the field is already present in the transaction log using the contains method of the Log. A log is a collection of log entries:

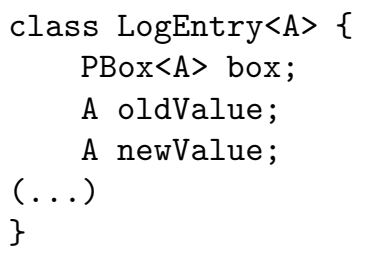

Each LogEntry represents the current state of a field during a transaction. It contains a reference to the field's PBox, the original content of the field (oldValue) and the current value of the field (newValue). If the log already contains an entry for fork then this entry is updated so its newValue field contains a reference to the argument of the set method. If the log contains no entry for fork a new entry is added where the oldValue contains the current content of fork and newValue is set to the argument of setFork.

getFork will first check if there is an entry for fork in the log. If so, it returns the current value of the newValue field of the entry, otherwise it adds a new entry to the log where both oldValue and newValue contain the current value in the fork field.

\subsection{The retry Method}

The retry method has a simple implementation:

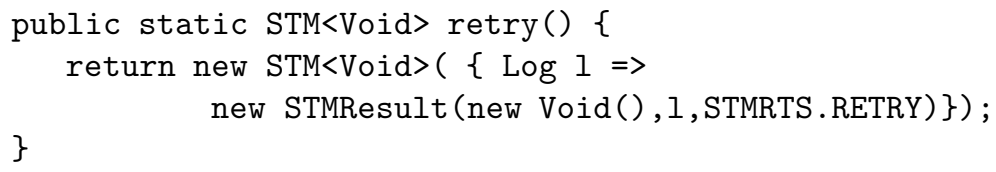

It simply stops the current transaction by changing the state of the transaction to STMRTS.RETRY. When a transaction returns STMRTS.RETRY the transaction is aborted (see the implementations for bind and then in Section 3.3).

The atomic method is responsible for restarting aborted transactions (Section 3.8).

\subsection{The orElse Method}

The orElse method is used to compose transactions as alternatives. To implement orElse, proper nested transactions are needed to isolate the execution of 
the two alternatives. If the first alternative calls retry, all the updates that it did must be invisible while executing the second alternative.

The orElse method is implemented in a similar way to orElse in STM Haskell. Each alternative is executed using a new log called nested log. All writes to fields are recorded in the nested log while reads must consult the nested $\log$ and the $\log$ for the enclosing transaction. If any of the alternatives finishes without retrying, its nested log is merged with the transaction log and a VALID STMResult is returned containing the merged $\log$. If the first alternative calls retry, then the second is executed using a new nested log. If the second alternative also calls retry, all three logs are merged and an STMResult containing the merged log and state set to RETRY is returned. All logs must be merged so that the aborted transaction will be executed again when one of the fields accessed in the transaction is modified by other transaction (Section 3.8). The reader should notice that, if both alternatives that retried modified the same filed of a TObject, the merged log will contain only one entry for that field. That does not matter as the merged $\log$ will be never used for committing, since the transaction was aborted. The final $\log$ is used to decide when the transaction should be restarted (Section 3.8).

\subsection{The atomic Method}

An STM action can be executed by calling atomic:

public static $<\mathrm{A}>\mathrm{A}$ atomic $(\mathrm{STM}<\mathrm{A}>\mathrm{t})$

The atomic method takes as an argument a transaction and executes it atomically with respect to other concurrent calls to atomic in other threads.

In order to execute a transactional action, the atomic method generates a fresh Log and then invokes the transaction:

$$
\begin{aligned}
& \log 1=\text { STMRTS.generateNewLog }() ; \\
& \text { STMResult }<A>r=\text { t.stm.invoke }(1) ;
\end{aligned}
$$

As described before, an invoked transaction will return a STMResult containing the result of executing the transaction, a log and the state of the transaction that can be either VALID or RETRY. A Log contains, besides log entries, a global lock that is used by atomic to update shared data structures when committing.

If the state of the executed transaction is RETRY, atomic acquires the global lock and validates the log of the transaction. To validate a log, each log entry is checked to see if its oldValue contains the same object that is currently in the field of the TObject. The current content of a field is accessed using the PBox in the log entry. If the log is invalid, the transaction is automatically restarted with a fresh log. If the log is valid, the transaction is only restarted once at least one of the fields that it accessed is modified by another thread. To do that, atomic first creates a SyncObject for the transaction. A SyncObject has two methods:

public void block();

public void unblock(); 
When a thread calls the block method of a SyncObject, the thread will block until some other thread calls the unblock method of the same object. If a thread calls unblock, and the blocked thread was already unblocked, the method simply returns with no effect. If a thread calls unblock before block was called, the thread waits for the call to block before continuing. Every PBox also contains a list of SyncObjects for the threads waiting for that field to be updated. After creating the SyncObject, it is added to the list of blocked threads in every PBox present in the transaction log. Then the transaction releases the global lock and calls the block method of the SyncObject, suspending the transaction. When the transaction is awaken, meaning that at least one of the Tobjects in its log was update, it will be executed again with a fresh log.

If the state of the transaction executed by atomic is VALID, atomic acquires the global lock and validates the transaction log. If valid, the transaction commits by modifying all TObjects with the content of its log. It also awakes all threads waiting for the commit. This is done by calling the unblock method of the SyncObjects contained in the PBoxes in the log. If the transaction log is invalid, the $\log$ is abandoned and the transaction is executed again with a fresh log.

\section{Related Work}

Transactional memory was initially studied as a hardware architecture [1023.5. Software transactional memory [25] is the idea of implementing all transactional semantics in software. Most works on STM in Java provide low level libraries to implement transactions 9817. Harris and Fraser [6] provide the first language with constructs for transactions. Their Java extension gives an efficient implementation of Hoare's conditional critical regions [1] through transactions, but transactions could not be easily composed. The Atomos language 4] is a Java extension that supports transactions through atomic blocks and also the retry construct to block transactions. Transactions are supported by the Transactional Coherence and Consistency hardware transactional memory model (TCC) [18, and programs are run on a simulator that implements the (TCC) architecture. No support composing transactions as alternatives is given.

CMTJava builds on work done in the Haskell language, by bringing the idea of composable memory transactions into an object oriented context. STM Haskell [7, is a new concurrency model for Haskell based on STM. Programmers define transactional variables (TVars) that can be read and written using two primitives:

readTVar : : TVar a $\rightarrow$ STM a

writeTVar : : Tvar a $\rightarrow$ a $\rightarrow$ STM a

The readTVar primitive takes a TVar as an argument and returns an STM action that, when executed, returns the current value of the TVar. The writeTVar primitive is used to write a new value into a TVar. In CMTJava, each field of a transactional object works as a TVar and each field has its get/set method that work just like readTVar and writeTVar. In STM Haskell, STM actions can be composed together using the same do notation used to compose IO actions in Haskell: 


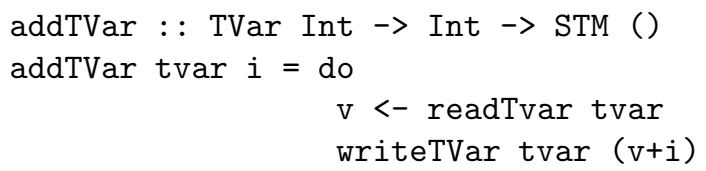

STM blocks in CMTJava are just an implementation of the do notation available in Haskell. The STM system described here is very similar to the one described in the STM Haskell paper, the main difference being that STM Haskell is implemented to run on uniprocessors. This simplifies the implementation as all calls to the $\mathrm{C}$ functions that support transactions in the runtime system are run without interruption. The simple implementation of CMTJava given in this paper can be executed on multi-core machines, as we use a global lock to avoid races during commits, at the cost of a huge bottleneck imposed by the lock.

In [12, an implementation of composable memory transactions using pure Haskell is given. CMTJava uses an implementation of the STM monad that is very similar to the one described in this paper, although CMTJava's implementation of the low level mechanisms to support transactions is very different, as it is based on the STM Haskell runtime system.

\section{Conclusions and Future Work}

In this paper we have presented CMTJava, a domain specific language for composable memory transactions in Java, in the style of STM Haskell. CMTJava guarantees that fields of transactional objects will only be accessed inside transactions. CMTJava is translated to pure Java + closures, that are supported by the BBGA Java extension [2]. Although the system was implemented in Java, the ideas could also be applied to any object oriented language that supports closures.

There are many directions for future work. A semantics for the language could be provided by extending Feather Weight Java [14] with the semantics for composable memory transactions in Haskell. Exceptions inside an atomic call could be handled in the same way as in STM Haskell.

The prototype implementation of the STM system presented in this paper is very naive and is given only to illustrate the concepts presented and as a proof of concept. Every time a field of a TObject is accessed the system must search in an array of LogEntries for the right entry before using the content of the field. The single lock used for committing transactions is also a bottleneck in the system. We plan to re-implement CMTJava in the future using a low level library for transactions in Java (e.g., [8]) or using other optimized technique for the implementation of transactional memory (a survey on the subject is given in [15]).

The source code for CMTJava can be obtained by contacting the authors.

Acknowledgment. The authors would like to thank $\mathrm{CNPq} /$ Brazil for the financial support provided. 


\section{References}

1. All About Monads. WWW page (December 2008), http://www.haskell.org/all_about_monads/html/index.html

2. Java Closures. WWW page (December 2008), http://www.javac.info/

3. JSR Proposal: Closures for Java. WWW page (December 2008), http://www.javac.info/consensus-closures-jsr.html

4. Carlstrom, B.D., McDonald, A., Chafi, H., Chung, J., Minh, C.C., Kozyrakis, C., Olukotun, K.: The ATOMOS transactional programming language. ACM SIGPLAN Notices 41(6), 1-13 (2006)

5. Hammond, L., Wong, V., Chen, M., Carlstrom, B.D., Davis, J.D., Hertzberg, B., Prabhu, M.K., Wijaya, H., Kozyrakis, C., Olukotun, K.: Transactional memory coherence and consistency, New York, NY, USA, vol. 32, p. 102. ACM, New York (2004)

6. Harris, T., Fraser, K.: Language support for lightweight transactions. ACM SIGPLAN Notices 38(11), 388-402 (2003)

7. Harris, T., Marlow, S., Peyton Jones, S., Herlihy, M.: Composable memory transactions. In: PPoPP 2005. ACM Press, New York (2005)

8. Herlihy, M., Luchangco, V., Moir, M.: A flexible framework for implementing software transactional memory. SPNOTICES: ACM SIGPLAN Notices 41 (2006)

9. Herlihy, M., Luchangco, V., Moir, M., Scherer III, W.N.: Software transactional memory for dynamic-sized data structures. In: PODC: 22th ACM SIGACTSIGOPS Symposium on Principles of Distributed Computing (2003)

10. Herlihy, M., Moss, J.E.B.: Transactional memory: Architectural support for lockfree data structures. In: Proceedings of the Twentieth Annual International Symposium on Computer Architecture (1993)

11. Hoare, C.A.R.: Towards a theory of parallel programming. Operating System Techniques, 61-71 (1972)

12. Huch, F., Kupke, F.: A high-level implementation of composable memory transactions in concurrent haskell. In: Butterfield, A., Grelck, C., Huch, F. (eds.) IFL 2005. LNCS, vol. 4015, pp. 124-141. Springer, Heidelberg (2006)

13. Hutton, G., Meijer, E.: Monadic Parsing in Haskell. Journal of Functional Programming 8(4), 437-444 (1998)

14. Igarashi, A., Pierce, B., Wadler, P.: Featherweight Java: A minimal core calculus for Java and GJ. TOPLAS 23(3), 396-459 (2001)

15. Larus, J., Rajwar, R.: Transactional Memory. Morgan \& Claypool (2006)

16. Lee, E.A.: The problem with threads. IEEE Computer 39(5), 33-42 (2006)

17. Marathe, V.J., Spear, M.F., Heriot, C., Acharya, A., Eisenstat, D., Scherer III, W.N., Scott, M.L.: Lowering the overhead of nonblocking software transactional memory. revised, University of Rochester, Computer Science Department (May 2006)

18. McDonald, A., Chung, J., Chafi, H., Minh, C.C., Carlstrom, B.D., Hammond, L., Kozyrakis, C., Olukotun, K.: Characterization of TCC on chip-multiprocessors. In: Proc. 14th International Conference on Parallel Architecture and Compilation Techniques (14th PACT 2005), pp. 63-74. IEEE Computer Society, Los Alamitos (2005)

19. Peterson, J., Hudak, P., Elliott, C.: Lambda in motion: Controlling robots with haskell. In: Gupta, G. (ed.) PADL 1999. LNCS, vol. 1551, pp. 91-105. Springer, Heidelberg (1999) 
20. Peyton Jones, S.: Tackling the awkward squad: monadic input/output, concurrency, exceptions, and foreign-language calls in Haskell. In: Engineering theories of software construction, pp. 47-96. IOS Press, Amsterdam (2001)

21. Peyton Jones, S.: Haskell 98 language and libraries: the revised report. Journal of Functional Programming 1(13) (2003)

22. Peyton Jones, S.: Beautiful Concurrency. O'Reilly, Sebastopol (2007)

23. Rajwar, R., Herlihy, M., Lai, K.K.: Virtualizing transactional memory. In: ISCA, pp. 494-505. IEEE Computer Society, Los Alamitos (2005)

24. Rajwar, R., James, G.: Transactional execution: Toward reliable, high-performance multithreading. IEEE Micro. 23(6), 117-125 (2003)

25. Shavit, N., Touitou, D.: Software transactional memory. DISTCOMP: Distributed Computing, 10 (1997)

\section{Appendix A: Translation Rules for TObjects}

Translation rules for TObjects are given as Haskell functions. These functions take as arguments Strings (i.e., names of variables and their types) and return as result a String representing the code to be generated. Strings in Haskell are represented using double quotes and the $(++)$ operation is used to concatenate two strings.

PBox objects are generated using the genPBox function:

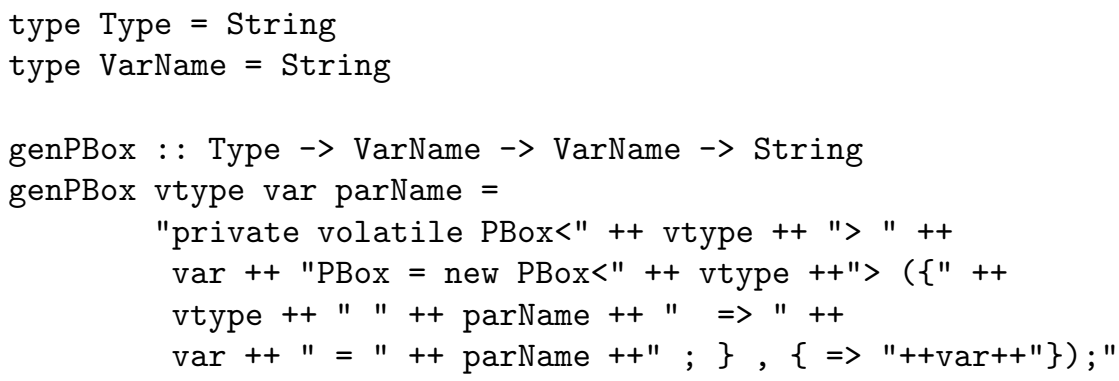

The parameters for genPBox are the type of the field of the transactional object (vtype), the name of the field var, and a random name for the parameters of the closures being defined parName.

Set methods for TObjects are generated using genSetMethod:

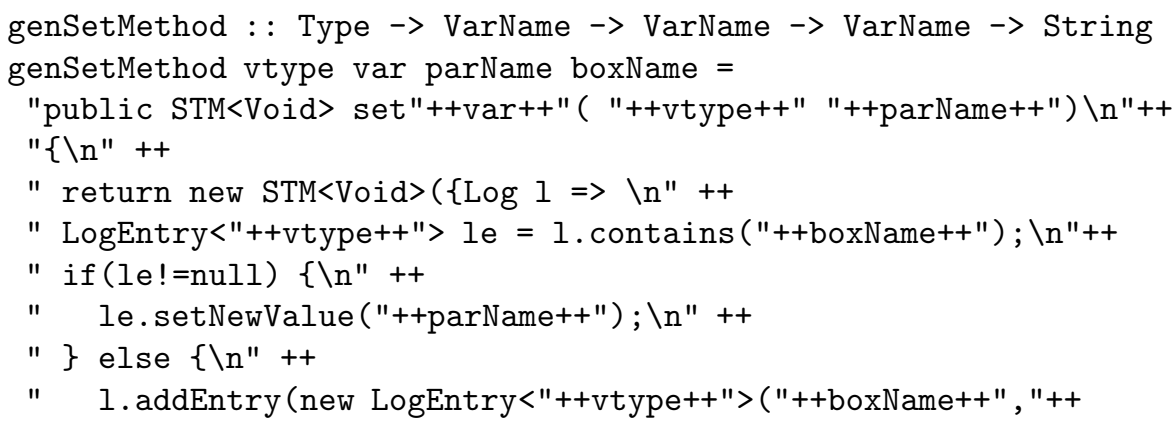




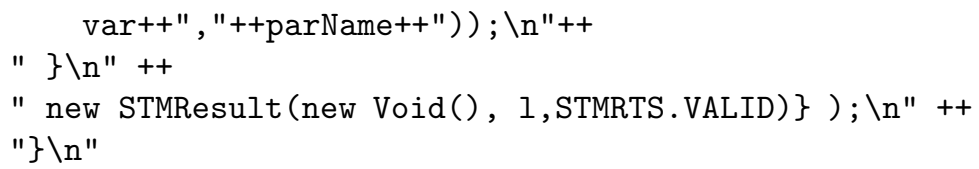

Get methods for TObjects are generated using genGetMethod:

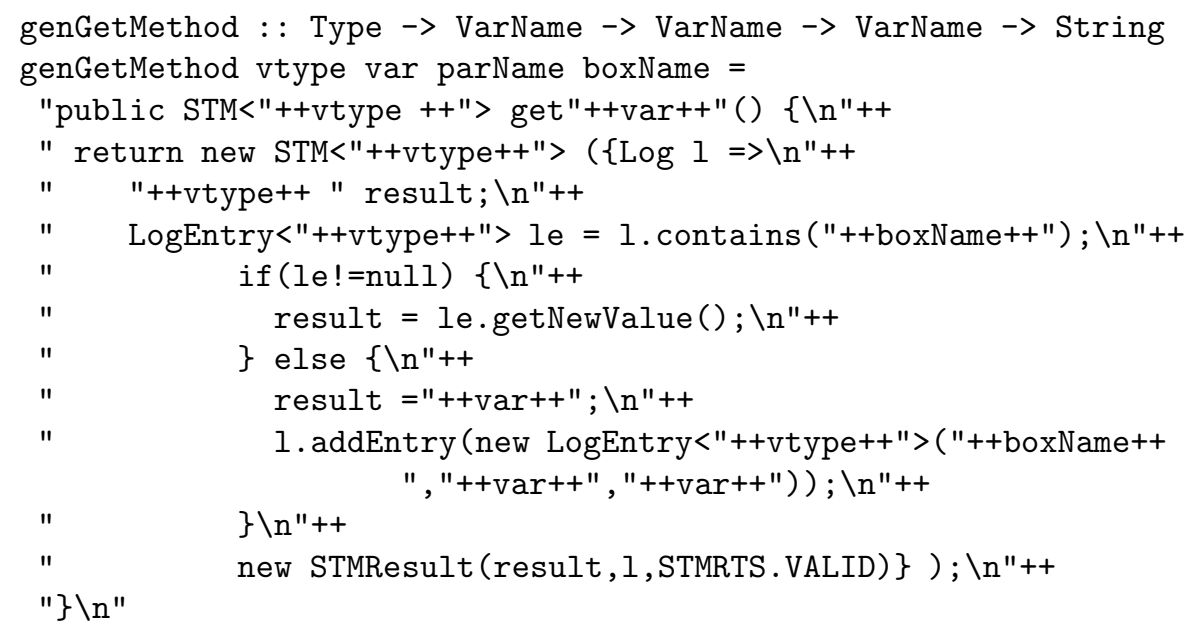

